\title{
Instabilities in Thermal Gravity with a Cosmological Constant
}

\author{
F. T. Brandt and J. Frenkel \\ Instituto de Física, Universidade de São Paulo, 05508-090, São Paulo, SP, BRAZIL
}

\begin{abstract}
It is shown that in quantum gravity at finite temperature, the effective potential evaluated in the tadpole approximation can have a local minimum below a certain critical temperature. However, when the leading higher order thermal loop corrections are included, one finds that no static solution exists at high temperature.
\end{abstract}

PACS numbers: 04.60.-m,11.10.Wx

\section{INTRODUCTION}

The purely attractive nature of gravity is the source of many instabilities, both at the classical and quantum levels. Instabilities in quantum gravity at finite temperature have been studied from various points of view 1, 2, 2, 3, 4, 5, 6, 7, 8]. Here we will consider this aspect in the spirit discussed by several authors $9,10,11,12$ in the context of the $\left(\phi^{3}\right)_{6}$ scalar model.

It is well known that at zero temperature, a gravitational system which would naturally fall upon itself, may remain in a static and uniform state in the presence of a cosmological constant. It may therefore be interesting to inquire whether such a situation can hold also at non-zero temperature. In this note, we consider the leading high temperature contributions to the effective potential in quantum gravity, assuming that self-interactions of the thermal matter are negligible. We show that, in this regime, the effective potential has no static solution. In the approximation where loops appear only through tadpole (one-point) diagrams we find that, for negative values of the cosmological constant $\Lambda$, the effective potential can have a local minimum below a critical temperature $T_{\text {cr }}$. These features are somewhat similar to the ones encountered in the $\left(\phi^{3}\right)_{6}$ scalar model at finite temperature [12]. But, in contrast with this theory, quantum gravity has a gauge invariance which requires that loops with any number of external lines must have the same $T^{4}$ leading dependence at high temperature [5, 6, 7]. When all such loop contributions are taken into account, it turns out that the effective potential exhibits instabilities at high temperatures. The terms which destabilize the local minimum of the effective potential are those associated with the hard thermal self-energy and vertex corrections to the tree amplitudes which join together the tadpole graphs. Such an instability indicates that, even in the presence of a cosmological constant, it is likely that a stable gravitational system cannot be formed at high temperature.

In section 2 we study the effective potential at finite temperature in the tadpole approximation, while in section 3 we discuss the effect of the inclusion of higher order thermal loop corrections. Some results which follow from this analysis may also be understood by means of a diagrammatic approach.

\section{EFFECTIVE POTENTIAL IN THE TADPOLE APPROXIMATION}

The effective potential $V_{\text {eff }}$, which is associated with configurations involving constant fields, is a useful quantity for studying the stability of a thermodynamic system 13, 14, 15, 16, 17]. In particular, the vacuum expectation values of the fields may be determined by minimizing $V_{\text {eff }}$. Since the Ricci scalar vanishes for constant fields, we can write the effective potential in the form

$$
V_{\text {eff }}=\frac{\Lambda}{8 \pi G} \sqrt{-g}+\mathcal{H},
$$

where $\mathcal{H}$ describes the matter fields coupled to gravity as well as the hard thermal effects.

In this section we shall consider the effective potential in the tadpole approximation, which is obtained by keeping in $\mathcal{H}$ only the linear term in the gravitational field. Then, one may express the effective potential as

$$
V_{\text {eff }}=\frac{\Lambda}{8 \pi G} \sqrt{-g}+\frac{1}{2} T_{0}^{\mu \nu} g_{\mu \nu}
$$

where $T_{0}^{\mu \nu}$ denotes the energy-momentum tensor of the system which includes the leading thermal contributions to lowest order. For example, in the presence of a constant scalar field $\phi_{c}$ with mass $m, T_{0}^{\mu \nu}$ can be written in the rest frame of the thermal bath as

$$
T_{0}^{\mu \nu}=\frac{m^{2} \phi_{c}^{2}}{2} \eta^{\mu \nu}+\omega \frac{\pi^{2} T^{4}}{90}\left(4 \eta^{\mu 0} \eta^{\nu 0}-\eta^{\mu \nu}\right),
$$

where $\omega$ is a numerical factor which depends on the quantum numbers of the thermal fields.

The above effective potential will have a local minimum at

$$
2 \frac{\delta V_{\text {eff }}}{\delta g_{\mu \nu}}=\frac{\Lambda}{8 \pi G} \sqrt{-g} g^{\mu \nu}+T_{0}^{\mu \nu}=0 .
$$

This equation can be solved in closed form and the solution, which corresponds to the vacuum expectation value $\left\langle g^{\mu \nu}\right\rangle$, is given by

$$
\left\langle g^{\mu \nu}\right\rangle=-\frac{\Lambda}{8 \pi G} \frac{T_{0}^{\mu \nu}}{\sqrt{-\operatorname{det} T_{0}}} .
$$

One can give a simple diagrammatic interpretation of this result, which may illustrate some points. To this end, let 
us define

$$
g_{\mu \nu}=\eta_{\mu \nu}+\kappa h_{\mu \nu}, \quad \kappa \equiv \sqrt{32 \pi G}
$$

and expand $V_{\text {eff }}$ as a power series in the field $h_{\mu \nu}$. Then, up to constants, we can write $V_{\text {eff }}$ in the form

$$
\begin{aligned}
V_{\mathrm{eff}} & =\frac{\kappa}{2} \bar{T}_{0}^{\mu \nu} h_{\mu \nu}+\frac{1}{2} h_{\mu \nu} D^{-1 \mu \nu ; \alpha \beta} h_{\alpha \beta} \\
& +\frac{\kappa}{3 !} \Gamma_{3}^{\mu \nu ; \alpha \beta ; \rho \sigma} h_{\mu \nu} h_{\alpha \beta} h_{\rho \sigma} \\
& +\frac{\kappa^{2}}{4 !} \Gamma_{4}^{\mu \nu ; \alpha \beta ; \rho \sigma ; \lambda \delta} h_{\mu \nu} h_{\alpha \beta} h_{\rho \sigma} h_{\lambda \delta}+\cdots .
\end{aligned}
$$

Here $\bar{T}_{0}^{\mu \nu}$ denotes the effective energy-momentum tensor

$$
\bar{T}_{0}^{\mu \nu} \equiv T_{0}^{\mu \nu}+\frac{\Lambda}{8 \pi G} \eta^{\mu \nu} .
$$

$D^{-1 \mu \nu ; \alpha \beta}$ is the inverse of the graviton propagator and $\Gamma_{3}, \Gamma_{4}, \cdots$ represent the graviton interaction vertices at zero momenta. Then, the Euler-Lagrange equation (4) can be written as

$$
\begin{gathered}
D^{-1 \mu \nu ; \alpha \beta} h_{\alpha \beta}+\frac{\kappa}{2 !} \Gamma_{3}^{\mu \nu ; \alpha \beta ; \rho \sigma} h_{\alpha \beta} h_{\rho \sigma}+ \\
+\frac{\kappa^{2}}{3 !} \Gamma_{4}^{\mu \nu ; \alpha \beta ; \rho \sigma ; \lambda \delta} h_{\alpha \beta} h_{\rho \sigma} h_{\lambda \delta}+\cdots=-\frac{1}{2} \kappa \bar{T}_{0}^{\mu \nu} .
\end{gathered}
$$

Using the Dyson-Schwinger method, we can now obtain a perturbative solution of (9) as a power series in $\bar{T}_{0}$, which may be represented graphically as

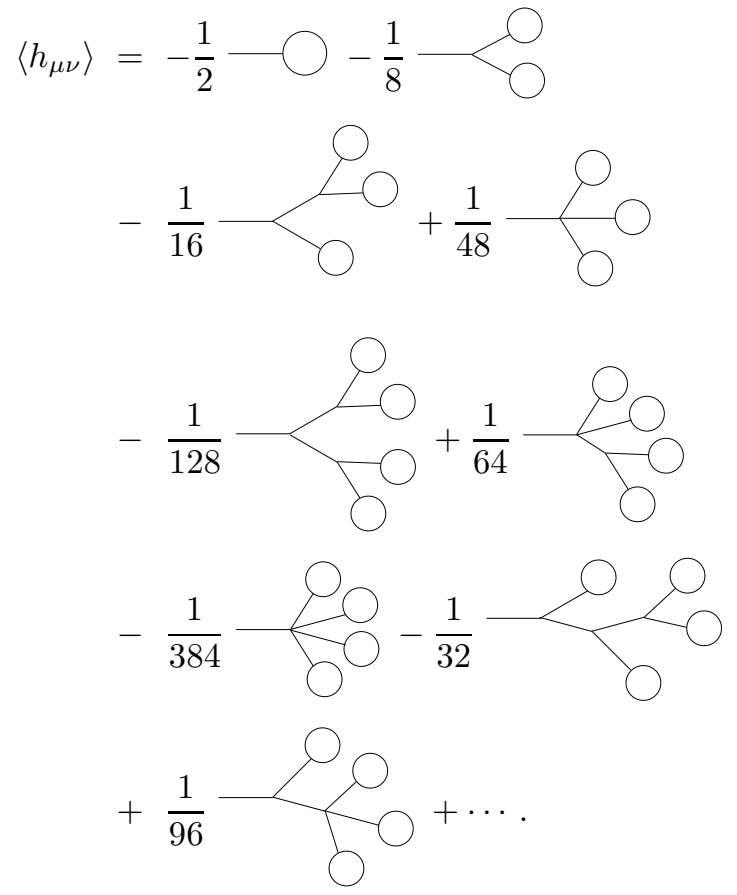

Here the blob corresponds to the tadpole $\bar{T}_{0}$ and each line represents the graviton propagator at zero momenta

$$
\begin{aligned}
D_{\mu \nu ; \alpha \beta} & =\frac{1}{2 \Lambda} \frac{\eta_{\mu \alpha} \eta_{\nu \beta}+\eta_{\mu \beta} \eta_{\nu \alpha}-\eta_{\mu \nu} \eta_{\alpha \beta}}{2} \\
& \equiv \frac{1}{2 \Lambda} P_{\mu \nu ; \alpha \beta} .
\end{aligned}
$$

One can verify, with the help of Eq. (8), that by summing the series of tree diagrams in Eq. (10), one arrives at a result which is equivalent to the one given by Eq. (5).

The effective potential (2) can have a local extremum at the value given by Eq. (5), provided $\left\langle g^{\mu \nu}\right\rangle$ is a real quantity. This condition requires that $\operatorname{det} T_{0}<0$ which entails, together with Eq. (3), the relation

$$
T<T_{\mathrm{cr}}=\left(\frac{45 m^{2} \phi_{c}^{2}}{\omega \pi^{2}}\right)^{1 / 4}
$$

This result, which is reminiscent of the one found in $\left(\phi^{3}\right)_{6}$ theory [12], indicates the existence of a critical temperature above which the effective potential (2) has no extrema.

Moreover, in order for a local minimum of the effective potential to exist, it is also necessary that

$$
\left.P_{\mu \nu ; \alpha \beta} \frac{\delta^{2} V^{\mathrm{eff}}}{\delta g_{\mu \nu} \delta g_{\alpha \beta}}\right|_{g^{\mu \nu}=\left\langle g^{\mu \nu}\right\rangle}>0,
$$

where $P_{\mu \nu ; \alpha \beta}$ is the projection operator introduced in Eq. (11). After a straightforward algebra, one finds that this requirement leads to the relation

$$
\Lambda\left[\left(\frac{\omega \pi^{2} T^{4}}{15}\right)^{2}-15 m^{4} \phi_{c}^{4}\right]>0 .
$$

In view of (12), this inequality implies that $\Lambda$ must be a negative quantity, in order to ensure the existence of a local minimum of the effective potential in the tadpole approximation.

We mention here that the constant vacuum expectation value of the scalar field may be obtained, as discussed for instance in ref. [12], through the introduction of selfinteractions between the scalar fields. Such expectation values would then correspond to the value which minimize the corresponding effective potential. In this note, for simplicity, we do not consider these self-interactions, since their inclusion would not alter the essence of the above considerations.

\section{HIGHER ORDER CONTRIBUTIONS TO THE EFFECTIVE POTENTIAL}

In quantum gravity, the leading contributions at high temperature satisfy simple Ward identities which reflect the gauge invariance of the theory [5, 6, 7]. These identities relate recursively all higher order Green functions to the one-point (tadpole) function. As a result, any $n$ point Green functions will exhibit the same leading $T^{4}$ dependence as does the tadpole diagram. All these thermal Green functions can be systematically generated, in the static case, by the effective action

$$
S_{\mathrm{th}}=\frac{\omega \pi^{2} T^{4}}{90} \int \mathrm{d}^{4} x \frac{\sqrt{-g}}{g_{00}^{2}}
$$


which is gauge invariant under static gauge transformations.

For consistency, all such higher order terms must be included in the effective potential (11), in which case $\mathcal{H}$ may be written for collisionless thermal matter, as

$$
\mathcal{H}=\frac{m^{2} \phi_{c}^{2}}{2} \sqrt{-g}-\frac{\omega \pi^{2} T^{4}}{90} \frac{\sqrt{-g}}{g_{00}^{2}} .
$$

We emphasize that the collisionless approximation may hold at temperatures such that $G T^{2} \ll 1$, but would not be justified for temperatures at the Planck scale, where collisions in the thermal matter become very important. Then, the Euler-Lagrange equation derived from this effective potential may be written as

$$
\frac{\Lambda}{8 \pi G} g^{\mu \nu}=-T^{\mu \nu}
$$

where the complete thermal energy-momentum tensor is given by

$$
\begin{aligned}
T^{\mu \nu} & =\frac{2}{\sqrt{-g}} \frac{\delta}{\delta g_{\mu \nu}} \int \mathrm{d}^{4} x \mathcal{H}=\frac{m^{2} \phi_{c}^{2}}{2} g^{\mu \nu} \\
& +\frac{\omega \pi^{2} T^{4}}{90} \frac{4 \delta_{0}^{\mu} \delta_{0}^{\nu}-g_{00} g^{\mu \nu}}{\left(g_{00}\right)^{3}} .
\end{aligned}
$$

We note that the thermal part of the tensor $T^{\mu \nu}$ is a traceless function at high temperature. Thus, by taking the trace of Eq. (17), one would get that

$$
\Lambda=-4 \pi G m^{2} \phi_{c}^{2} .
$$

In view of this condition, we see that Eq. (17) cannot have a consistent solution $\left\langle g^{\mu \nu}\right\rangle$ at nonzero temperature. Consequently, we may conclude that the complete thermal effective action does not have a local minimum.

This behavior may also be seen from a diagrammatic approach similar to the one which led to Eq. (10). In the present case, one finds that the vacuum expectation value of the gravitational field can be related to a series of diagrams of the form

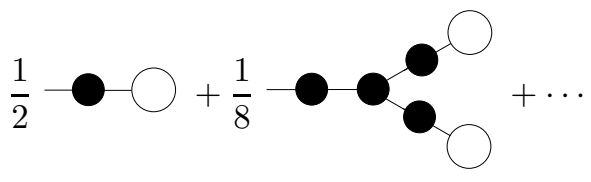

where the full blobs denote the hard thermal self-energy and vertex corrections to the tadpole tree-amplitudes. But it may be checked that the sum of the above series of diagrams would lead to a divergent result, so that a regular solution $\left\langle g^{\mu \nu}\right\rangle$ does not exist in this case.

The fact that higher order thermal corrections destabilize the local minimum of the effective potential may be understood on physical grounds. This is because such contributions lead to the appearance of an imaginary part in the free-energy, which can be related to the decay rate of the quantum vacuum. Work on this aspect is in progress.

\section{Acknowledgments}

We would like to thank Professors Ashok Das and J. C. Taylor for helpful discussions. We are grateful to $\mathrm{CNPq}$ and FAPESP, Brazil, for financial support.
[1] D. J. Gross, M. J. Perry, L. G. Yaffe, Phys. Rev. D25 (1982) 330.

[2] Y. Kikuchi, T. Moriya, H. Tsukahara, Phys. Rev. D29 (1984) 2220.

[3] N. Nakazawa, T. Fukuyama, Nucl. Phys. B252 (1985) 621.

[4] P. S. Gribosky, J. F. Donoghue, B. R. Holstein, Ann. Phys. 190 (1989) 149.

[5] J. Frenkel, J. C. Taylor, Z. Phys. C49 (1991) 515.

[6] A. Rebhan, Nucl. Phys. B351 (1991) 706.

[7] F. T. Brandt, J. Frenkel, J. C. Taylor, Nucl. Phys. B374 (1992) 169.

[8] J. D. Barrow, G. F. R. Ellis, R. Maartens and C. G. Tsagas, Class. Quant. Grav. 20 (2003) L155.
[9] M. Le Bellac, D. Poizat, Z. Phys. C47 (1990) 125.

[10] T. Grandou, M. Le Bellac, D. Poizat, Phys. Lett. B249 (1990) 478.

[11] R. D. Pisarski, Nucl. Phys. A525 (1991) 175.

[12] T. Altherr, T. Grandou, R. D. Pisarski, Phys. Lett. B271 (1991) 183.

[13] S. R. Coleman, E. Weinberg, Phys. Rev. D7 (1973) 1888.

[14] L. Dolan, R. Jackiw, Phys. Rev. D9 (1974) 3320.

[15] S. Weinberg, Phys. Rev. D9 (1974) 3357.

[16] Y. Fujimoto, R. Grigjanis, R. Kobes, Prog. Theor. Phys. 73 (1985) 434.

[17] T. S. Evans, Z. Phys. C36 (1987) 153. 